\title{
Caracterización comercial y nutricional de la grasa subcutánea de terneros y vacas de desvieje sacrificados en la Comunidad Autónoma del País Vasco
}

\author{
Aurtenetxe, M. '; Belaunzaran, X. '; Bravo-Lamas, L. ' ; Gamarra, D. ${ }^{\text {, }}$ Barron, L.J.R. ${ }^{1}$ y Aldai, N. ${ }^{@}$
}

'Grupo de Investigación Lactiker. Departamento de Farmacia y Ciencias de los Alimentos. Universidad del País Vasco/ Euskal Herriko Unibertsitatea (UPV/EHU). Vitoria-Gasteiz. España.

${ }^{2}$ Grupo de Investigación Biomics. Departamento de Zoología y Biología Celular. Universidad del País Vasco/ Euskal Herriko Unibertsitatea (UPV/EHU). Vitoria-Gasteiz. España.

\section{PALABRAS CLAVE ADICIONALES}

Caracterización comercial.

Terneros.

Vacas de desvieje.

Grasa subcutánea.

Ácidos grasos.

Isómeros trans.

\section{RESUMEN}

Este trabajo se ha llevado a cabo con el objetivo de comparar la calidad comercial de la canal y la composición de ácidos grasos de la grasa subcutánea de vacas de desvieje de raza Frisona de más de 30 meses de edad, y de terneros de 12 meses de edad de las razas Pirenaica y Salers sacrificados en un matadero comercial de la Comunidad Autónoma del País Vasco. Las vacas de raza Frisona presentaron canales de menor peso $(p<0,05)$, aunque similar al grupo de los terneros de raza Pirenaica y menor conformación $(p<0,001)$ y estado de engrasamiento $(p<0,01)$ en comparación con los grupos de terneros. La grasa subcutánea de las vacas de desvieje presentó un mayor contenido de ácidos grasos saturados $(p<0,001)$ y ramificados $(p<0,05)$, y un menor contenido de ácidos grasos monoinsaturados $(p<0,01)$ y poliinsaturados $(p<0,001)$ en comparación con la de los terneros de las razas Pirenaica y Salers. El porcentaje de ácidos grasos monoinsaturados de tipo trans fue significativamente superior en la grasa subcutánea de los terneros de raza Pirenaica $(p<0,001)$, mientras que el contenido de 10t-1 8:1 fue significativamente mayor en la grasa de terneros de raza Pirenaica, intermedio en la grasa de terneros de raza Salers, y menor en la grasa de las vacas de desvieje $(p<0,001)$. Esto dio lugar a una relación $10 t / 11 t<1$ y un perfil isomérico relativo de trans-18:1 y ácidos linoleico conjugados más saludable en la grasa de las vacas de desvieje de raza Frisona en comparación con los grupos de terneros. Esta idea podría contribuir a la valorización de las canales procedentes de vacas de desvieje desde el punto de vista de su comercialización y de la rentabilidad de las explotaciones ganaderas de vacuno en la Comunidad Autónoma del País Vasco.

Commercial and nutritional characterization of the subcutaneous fat of calves and cows for culling slaughtered in the autonomous community of the Basque country

\section{SUMMARY}

The present work was undertaken to compare the commercial carcass quality and the fatty acid composition of subcutaneous fat from Friesian culled cows of over 30 months of age, and calves of 12 months of age from Pirenaica and Salers breeds slaughtered in a commercial abattoir in the Basque Country. Friesian culled cows provided lighter carcasses $(p<0.05)$, although similar to calves from Pirenaica breed, with lower conformation $(p<0.001)$ and less fat cover $(p<0.01)$ compared to other groups of calves. The subcutaneous fat of culled cows provided higher content of saturated $(p<0.001)$ and branched-chain fatty acids $(p<0,05)$, and lower content of monounsaturated $(p<0.01)$ and polyunsaturated fatty acids $(p<0,001)$ compared to calves from Pirenaica and Salers breeds. The percentage of trans-monounsaturated fatty acids was significantly higher in the subcutaneous fat of calves from Pirenaica breed $(p<0.001)$, while the 10*18:1 content was significantly higher in the fat of calves from Pirenaica breed, intermediate in the fat of calves from Salers breed, and lower in the fat of culled cows $(p<0.001)$. These provided a $10 t / 11$ t ratio $<1$ and a healthier relative trans-18:1 and conjugated linoleic acid isomeric profile in the fat of Friesian culled cows compared to other groups of calves, which may provide culled cow carcasses with a higher added value from the commercialization point of view and of the profitability of cattle farms in the Basque Country.

\section{INTRODUCCIÓN}

En España, los principales grupos comerciales bovinos destinados al consumo humano los forman la carne de ternera y de novillo/a (Alberti et al., 2005) mientras que, en general, los ingresos provenientes de vacas lecheras de desvieje vendidas para producción cárnica suponen alrededor del 5-10\% del total comercializado tal y como ocurre en la mayoría de los países europeos (Cabaraux et al., 2005; Bazzoli et al., 2014). Una vez en el matadero, las vacas de desvieje producen 
canales de escasa conformación y en la mayoría de los casos su carne se destina a la elaboración de derivados cárnicos, principalmente carne picada (Cranwell et al., 1996). Sin embargo, son muchos los autores que defienden las características sensoriales deseables de este tipo de carne (Jurie et al., 2007; Lizaso et al., 2011) y en países como Francia por ejemplo, el consumo de carne procedente de vacas de desvieje que anteriormente fueron destinadas a la producción de leche supone aproximadamente la mitad del consumo total de carne bovina (Jurie et al., 2007).

En la cornisa cantábrica, las explotaciones de ganado lechero (fundamentalmente de raza Frisona) son generalmente familiares, de pequeño tamaño y practican un manejo semi-extensivo. Después de una vida productiva intensa y cumplida su función lechera, las vacas son vendidas a empresas de carnización para satisfacer la demanda de establecimientos de restauración (sidrerías y restaurantes) de la zona norte, en general, y de la Comunidad Autónoma del País Vasco (CAPV) en particulat. Para evitar la gran heterogeneidad en las canales producidas, las vacas de desvieje se suelen acabar con cantidades variables de concentrado y ensilado, y cuando es posible también con hierba de calidad, con el objetivo final de mejorar la conformación y engrasamiento de las canales y las características físico-químicas y sensoriales de la carne, incrementando así el precio final del producto (Matulis et al., 1987; Carballo y Moreno, 2006). Por otra parte, las razas de aptitud marcadamente cárnica como pueden ser las razas Pirenaica y Salers son producidas principalmente bajo sistemas intensivos de alimentación.

En los últimos años, el interés del consumidor por la cantidad y la calidad nutricional de la grasa va en continuo aumento (Wood et al., 2008; Downs et al., 2013). Tanto la industria alimentaria como los organismos nacionales e internacionales relacionados con la alimentación y la salud pública vienen limitando el contenido de ácidos grasos (AG) de tipo trans de origen industrial en alimentos con alto contenido en grasa por su relación con el riesgo de padecer enfermedades cardiovasculares (Mozaffarian et al., 2009). Sin embargo, la carne y los productos cárnicos son alimentos que en la actualidad están en entredicho debido a que no todas las grasas tipo trans de origen animal, y en particular las de los rumiantes, presentan efectos adversos sobre la salud humana (Bauchart et al., 2007; Roy et al., 2007; Burnyeat, 2015). Como se ha constatado en varios trabajos de revisión (Aldai et al., 2013; Bessa et al., 2015) su perfil lipídico está marcadamente influenciado por la alimentación del animal.

El objetivo de este trabajo ha sido la caracterización comercial de las canales bovinas y la comparación de la calidad nutricional de la grasa subcutánea (grasa SC) a través del perfil de AG totales de terneros de razas cárnicas (Pirenaica y Salers de 1 año de edad) frente al de vacas de desvieje (de más de 3 partos) de raza Frisona. Estos animales han sido elegidos como representación de la actividad ganadera y comercial de la CAPV. El estudio pretende además aportar evidencias científicas que puedan contribuir a aumentar el valor añadido de la carne procedente de animales de desvieje.

\section{MATERIALES Y MÉTODOS}

\section{MUeSTREO}

En un matadero comercial de la CAPV (Urkaiko S. Coop., Zestoa, Gipuzkoa) se recogieron muestras de grasa SC de bovinos de diferentes razas sacrificados en días aleatorios durante los meses de junio y julio de 2014. Entre las 24 y 48 horas de sacrificio se recogieron un total de 152 muestras de grasa SC de la media canal izquierda, localizada entre la $5^{\mathrm{a}}$ y $6^{\mathrm{a}}$ costilla. Las muestras de grasa fueron transportadas al laboratorio en refrigeración, se envasaron al vacío y se conservaron a $-80^{\circ} \mathrm{C}$ hasta su posterior análisis. En el matadero comercial se obtuvo información de la raza, edad de sacrificio, peso de la canal y clasificación de la misma en cuanto a conformación (referida al desarrollo de los perfiles de la canal y, en particular, de las partes esenciales de la misma) y estado de engrasamiento (referido a la importancia de la grasa en el exterior de la canal y en la cara interna de la cavidad torácica), de acuerdo con la clasificación europea (RD 225/2008) pero adaptadas en cada caso a una escala cuantitativa (escala de 1 a 18 puntos para la conformación, y de 1 a 15 puntos para el estado de engrasamiento).

De las 152 muestras recogidas en el matadero, 115 fueron de terneros de raza Pirenaica, 13 de terneros de raza Salers y 24 de vacas de raza Frisona. Del total de muestras, se seleccionaron al azar 24 muestras de terneros de raza Pirenaica con un rango de edad similar (aproximadamente 1 año) a los de la raza Salers, con objeto de evitar una alta disparidad en el tamaño de los grupos de muestras. Los datos de edad de sacrificio y características de la canal de los tres grupos comerciales analizados bovinos se presentan en la tabla I.

\section{ANÁLISIS DE ÁCIDOS GRASOS TOTALES}

Para la determinación de los AG se liofilizaron 50 mg de grasa SC y, a continuación, se llevó a cabo una transmetilación directa con metóxido sódico (0,5 N) (Kramer et al., 1997) previa adición de $1 \mathrm{~mL}$ de patrón interno (23:0 metilado, Sigma-Aldrich, Madrid, España) disuelto en tolueno $(4 \mathrm{mg} / \mathrm{mL})$. Los AG metilados se analizaron en un equipo de cromatografía de gases con detector de ionización de llama (Modelo 7890A, Agilent, Madrid, España) utilizando separadamente dos columnas cromatográficas capilares de sílice fundida (Supelco, Bellefonte, EEUU). Por un lado, una columna SP-2560 (100 m x 0,25 mm d.i.; 0,2 $\mu \mathrm{m}$ de espesor de película) fue utilizada con dos programas de temperatura distintos; el primero para obtener el perfil total de AG, y el segundo para el análisis específico de los isómeros trans-18:1 y otros AG que coeluyen (Kramer et al. 2008). Por otro lado, una columna iónica SLB-IL111 (100 m x 0,25 mm d.i.; 0,2um de espesor de película) fue utilizada para la separación de los isómeros ácido linoleico conjugados (ALC) y de otros ácidos dienoicos y trienoicos que coeluyen en la columna SP-2560 (Delmonte et al., 2011). Las condiciones de análisis se han descrito previamente en Bravo-Lamas et al. (2016). La identificación de los AG de las muestras de grasa SC se realizó por comparación de los tiempos de retención de los picos cromatográficos con los de sustancias puras comerciales obtenidas de Nu-Check Prep, Inc. (Wa- 
Tabla I. Edad de sacrificio y parámetros de canal de los tres grupos comerciales de vacuno: terneros de razas Pirenaica y Salers; vacas de desvieje de raza Frisona en la Comunidad Autónoma del País Vasco (Age at slaughter and carcass traits from the three bovine commercial types: calves from Pirenaica and Salers breeds; Friesian cull cows in the Basque Autonomous Community).

\begin{tabular}{|c|c|c|c|c|c|}
\hline & Pirenaica $(n=24)$ & Salers $(n=13)$ & Frisona $(n=24)$ & e.e.m. & $\mathrm{p}$-valor \\
\hline Edad de sacrificio (meses) & $11,7^{b}(10,2-13,0)$ & $12,6^{b}(10,3-14,0)$ & $69,5^{\mathrm{a}}(31,2-112)$ & 3,95 & $<0,001$ \\
\hline Peso de la canal caliente $(\mathrm{kg})$ & $285^{\mathrm{b}}(173-361)$ & $331^{\mathrm{a}}(291-364)$ & $291^{\mathrm{b}}(194-405)$ & 6,44 & 0,020 \\
\hline Conformación* (1-18) & $10,4^{a}(8,00-13,0)$ & $8,46^{b}(8,00-10,0)$ & $3,13 c(1,00-6,00)$ & 0,452 & $<0,001$ \\
\hline Engrasamiento* (1-15) & $5,17^{\mathrm{a}}(2,00-7,00)$ & $5,69^{\mathrm{a}}(5,00-7,00)$ & $3,79^{b}(1,00-6,00)$ & 0,242 & 0,004 \\
\hline
\end{tabular}

$a, b, c$ Letras superíndices diferentes en una misma fila indican diferencias significativas entre los grupos comerciales ( $p<0,05)$. Valor medio (mín. - máx.); e.e.m., error estándar de la media.

*Conformación y estado de engrasamiento de acuerdo con la clasificación europea (BOE, 2008) pero adaptadas en cada caso a escala cuantitativa:

Conformación; 1=P-, 2=P, 3=P+, 4=O-, 5=O, 6=O+, 7=R-, 8=R, 9=R+, 10=U-, 11=U, 12=U+, 13=E-, 14=E, 15=E+, 16=S-, 17=S, 18=S+

Engrasamiento: $1=1-, 2=1,3=1+, 4=2-, 5=2,6=2+, 7=3-, 8=3,9=3+, 10=4-, 11=4,12=4+, 13=5-, 14=5,15=5+$.

terville, EEUU) Supelco (Bellefonte, EEUU), Matreya, LLC (State College, EEUU) y Larodan Fine Chemicals AB (Limhamn, Suecia). También se utilizó información cromatográfica de fracciones obtenidas a partir de la separación de los AG totales metilados mediante extracción en fase sólida con ión plata (Belaunzaran et al., 2014, 2016; Kramer et al., 2008), y por comparación con tiempos y orden de elución de AG descritos en la literatura científica (Alves y Bessa, 2009, 2014; CruzHernandez et al., 2004; Delmonte et al., 2011; Kramer et al., 2008). El análisis cuantitavo se llevó a cabo según la metodología de análisis previamente descrita en Bravo-Lamas et al. (2016), los resultados se han expresado como porcentaje de área normalizada respecto al total de AG.

\section{ANÁLISIS ESTADÍSTICO}

El análisis estadístico se llevó a cabo mediante el paquete estadístico IBM SPSS Statistics versión 22 (SPSS Inc, IBM Corporation, Nueva York, EEUU). Dada la naturaleza del estudio (muestreo comercial), se estudiaron las diferencias en las características comerciales de la canal y en la composición de AG de la grasa SC entre los grupos comerciales de ganado vacuno (terneros de las razas Pirenaica, y Salers, y vacas de raza Frisona). Se aplicó el análisis de la varianza de acuerdo al siguiente modelo lineal general:

$\mathrm{Y}_{i j}=\mu+\mathrm{GCB}_{i}+e_{i j}$ incluyendo el grupo comercial bovino (GCB) como factor fijo. Debido al tamaño desigual de los grupos a comparar, se realizó la prueba de Brown-Forsythe para evaluar el efecto del factor GCB sobre las variables analizadas, y se empleó el test de Games-Howell para el análisis de comparaciones múltiples. Se determinaron diferencias estadísticamente significativas cuando $\mathrm{p}<0,05$.

\section{RESULTADOS Y DISCUSIÓN}

La tabla I muestra los resultados de la comparación de las características de canal de los grupos comerciales bovinos sacrificados en un matadero comercial en la CAPV. Se observaron diferencias estadísticamente significativas entre los grupos comerciales en los parámetros de edad al sacrificio $(\mathrm{p}<0,001)$, peso de la canal $(\mathrm{p}<0,05)$, conformación $(\mathrm{p}<0,001)$ y estado de engrasamiento $(\mathrm{p}<0,01)$. Las vacas de desvieje de raza Frisona (69,5 meses de media) presentaron canales de menor peso aunque similar al grupo de los terneros de raza Pirenaica (valor medio para ambos grupos comerciales bovinos de $288 \mathrm{~kg}$ ), menor conformación $(3,13 ; p<0,001)$ y menor engrasamiento $(3,79 ; p<0,01)$ en comparación con los grupos de terneros. En general, los valores de conformación de las canales obtenidas a partir de las vacas de desvieje de raza Frisona en este estudio fueron similares a los obtenidos por Carballo y Moreno (2006) en vacas Frisonas de Galicia, mientras que los valores de engrasamiento fueron algo inferiores. Los terneros de la raza Salers, con una edad de sacrificio similar a los de la raza Pirenaica (valor medio para ambas razas de 12,2 meses), presentaron las canales de mayor peso $(331 \mathrm{~kg})$, y valores de conformación intermedia $(8,46)$ y de engrasamiento similares a los de los terneros de raza Pirenaica (valor medio para ambas razas de 5,43).

El perfil de AG totales de la grasa SC es un buen indicativo de los procesos de biohidrogenación ruminal debido a la mayor acumulación de metabolitos intermediarios tales como AG tipo trans en comparación con otros tejidos (Fritsche et al., 2001; Jerónimo et al., 2011). Por lo tanto, el análisis por metilación directa de la grasa SC permite obtener de forma rápida una visión general de la composición en AG de la grasa del animal. El perfil de AG de la grasa SC de los tres grupos comerciales bovinos se muestra en la tabla II. El contenido total de AG saturados (AGS) fue mayor en la grasa de las vacas de desvieje de raza Frisona $(51,4 \%)$ respecto a los terneros de las razas Pirenaica y Salers (valor medio para ambos grupos de terneros de $45,4 \%$; $p<0,001)$. La mayor acumulación de AGS en la grasa SC de las vacas de desvieje, aún con un menor engrasamiento de las canales, fue fundamentalmente debido a una mayor acumulación de los ácidos palmítico (16:0) y esteárico (18:0) lo cual podría deberse, por una parte, a la inclusión de pienso concentrado en la dieta de estos animales, o bien a que el alimento se mantiene durante más tiempo en el estómago de las vacas cuando éstas son alimentadas a base de pasto provocando un mayor grado de biohidrogenación de los AG poliinsaturados (AGP) ingeridos (Doreau y Ferlay, 1995; Glasser et al., 2008; Chillard et al., 2007). Además, un mayor tiempo del alimento en el estómago podría suponer, así mis- 
Tabla II. Composición de ácidos grasos (\% sobre el total de ácidos cuantificados) de la grasa subcutánea de los tres grupos comerciales de vacuno: terneros de razas Pirenaica y Salers; vacas de desvieje de raza Frisona en la Comunidad Autónoma del País Vasco (Fatty acid composition (\% of total fatty acids) of subcutaneous fat from the three bovine commercial types: calves from Pirenaica and Salers breeds; Friesian cull cows in the Basque Autonomous Community).

\begin{tabular}{|c|c|c|c|c|c|}
\hline & Pirenaica $(n=24)$ & Salers $(n=13)$ & Frisona $(n=24)$ & e.e.m. & $p$-valor \\
\hline AGS & $45,5^{\mathrm{b}}$ & $45,2^{b}$ & $51,4^{a}$ & 0,743 & 0,001 \\
\hline AGR & $1,52^{b}$ & $1,47^{\mathrm{b}}$ & $1,83^{a}$ & 0,0559 & 0,022 \\
\hline AGM & $48,1^{a}$ & $48,5^{a}$ & $43,7^{b}$ & 0,737 & 0,015 \\
\hline cis -AGM & 41,7 & 43,8 & 39,9 & 0,790 & 0,177 \\
\hline cis $-16: 1$ & 4,25 & 4,29 & 4,43 & 0,235 & 0,943 \\
\hline cis $-18: 1$ & $35,4^{a, b}$ & $37,3^{a}$ & $33,5^{\mathrm{b}}$ & 0,532 & 0,023 \\
\hline trans -AGM & $6,37^{a}$ & $4,64^{b}$ & $3,75^{b}$ & 0,248 & $<0,001$ \\
\hline trans $-16: 1$ & $0,196^{a}$ & $0,139^{b}$ & $0,155^{b}$ & 0,00707 & 0,003 \\
\hline trans $-18: 1$ & $6,18^{a}$ & $4,51^{b}$ & $3,60^{b}$ & 0,243 & $<0,001$ \\
\hline $10 t-$ & $2,88^{a}$ & $1,81^{b}$ & $0,437^{C}$ & 0,204 & $<0,001$ \\
\hline $11 t-$ & 1,07 & 0,942 & 1,25 & 0,0665 & 0,193 \\
\hline $10 t / 11 t$ & $3,87^{a}$ & $2,30^{a}$ & $0,400^{b}$ & 0,361 & $<0,001$ \\
\hline ALC & 0,605 & 0,546 & 0,513 & 0,0202 & 0,127 \\
\hline AGP & $3,38^{a}$ & $3,61^{a}$ & $1,81^{b}$ & 0,154 & $<0,001$ \\
\hline$n-6$ & $3,09^{a}$ & $3,35^{a}$ & $1,52^{b}$ & 0,151 & $<0,001$ \\
\hline$n-3$ & 0,272 & 0,248 & 0,271 & 0,00975 & 0,630 \\
\hline$n-6 / n-3$ & $11,6^{a}$ & $13,5^{a}$ & $5,95^{\mathrm{b}}$ & 0,526 & $<0,001$ \\
\hline AGP/AGS & $0,0747^{a}$ & $0,0805^{a}$ & $0,0352^{b}$ & 0,00367 & $<0,001$ \\
\hline
\end{tabular}

e.e.m.: error estándar de la media.

AGS: ácidos grasos saturados; AGR: ácidos grasos ramificados; AGM: ácidos grasos monoinsaturados; t: trans; ALC: ácidos linoleico conjugados; AGP: ácidos grasos poliinsaturados.

$a: b: c$ Letras superíndices diferentes en una misma fila indican diferencias significativas entre los grupos comerciales ( $p<0: 05)$.

mo, una mayor acumulación de acetato, dando lugar a mayor síntesis de AGS como el 16:0 (Kennedy et al., 1977). Se encontraron diferencias significativas en el porcentaje total de AG ramificados (AGR), siendo las vacas de desvieje las que presentaron mayor contenido $(1,83 \% ; \mathrm{p}<0,05)$ frente a los terneros (valor medio para los dos grupos de terneros de 1,49\%). Los AGR mayoritarios en los tres grupos comerciales bovinos fueron anteiso-17:0 e iso-17:0 (valor medio de 0,632\% y 0,289\%, respectivamente). Estos AGR son de origen microbiano y su síntesis y acumulación está relacionada con el empleo de isovalerato, isobutirato y 2-metil-butirato como sustratos metabólicos (Fievez et al., 2012).

La grasa SC de las canales de los terneros de las razas Salers y Pirenaica presentó un mayor porcentaje de AG monoinsaturados (AGM) (valor medio para ambas razas de 48,3\%) en comparación con el de las canales de las vacas de desvieje $(43,7 \% ; p<0,01)$. No se observaron diferencias significativas en los AGM tipo cis aunque sí que se encontraron diferencias en el contenido total de cis-18:1, principalmente debido al ácido oleico (9c18:1), siendo la grasa SC de los terneros de raza Salers la que acumuló mayor porcentaje (37,3\%) en comparación con la de las vacas $(33,5 \%)$, mientras que la grasa subcutánea de los terneros de raza Pirenaica presentó valores intermedios $(35,4 \% ; \mathrm{p}<0,05)$. Generalmente, una mayor disponibilidad metabólica de glucosa cuando el animal es alimentado a base de concentrado aumenta la expresión de enzimas lipogénicas, como la estearoil-CoA desaturasa, favoreciendo la acumulación de 9c-18:1 en el tejido graso (Smith et al., 2009). Algunas investigaciones indican preferencia de substrato de la esteroil-CoA desaturasa sobre los AG sintetizados de novo, lo cual podría explicar los mayores porcentajes de 9c-18:1 en la grasa SC de los animales alimentados a base de concentrado (Collins et al., 2010). Los resultados de un estudio previo llevado a cabo por Rosa et al. (2014) refuerzan esta teoría ya que se observó que, a medida que aumentaba el contenido de cereal en la ración de machos jóvenes de raza Frisona, aumentaba la proporción de 9c-18:1 y de AG trans, mientras que el contenido de los ácidos esteárico (18:0) y vaccénico (AV, 11t-18:1) disminuía. En el presente estudio, estos resultados se ven reflejados en la grasa SC procedente de los terneros (tabla II, figura 1) que posiblemente fueron cebados a base de pienso concentrado, práctica muy habitual en la producción de terneros en la CAPV. 


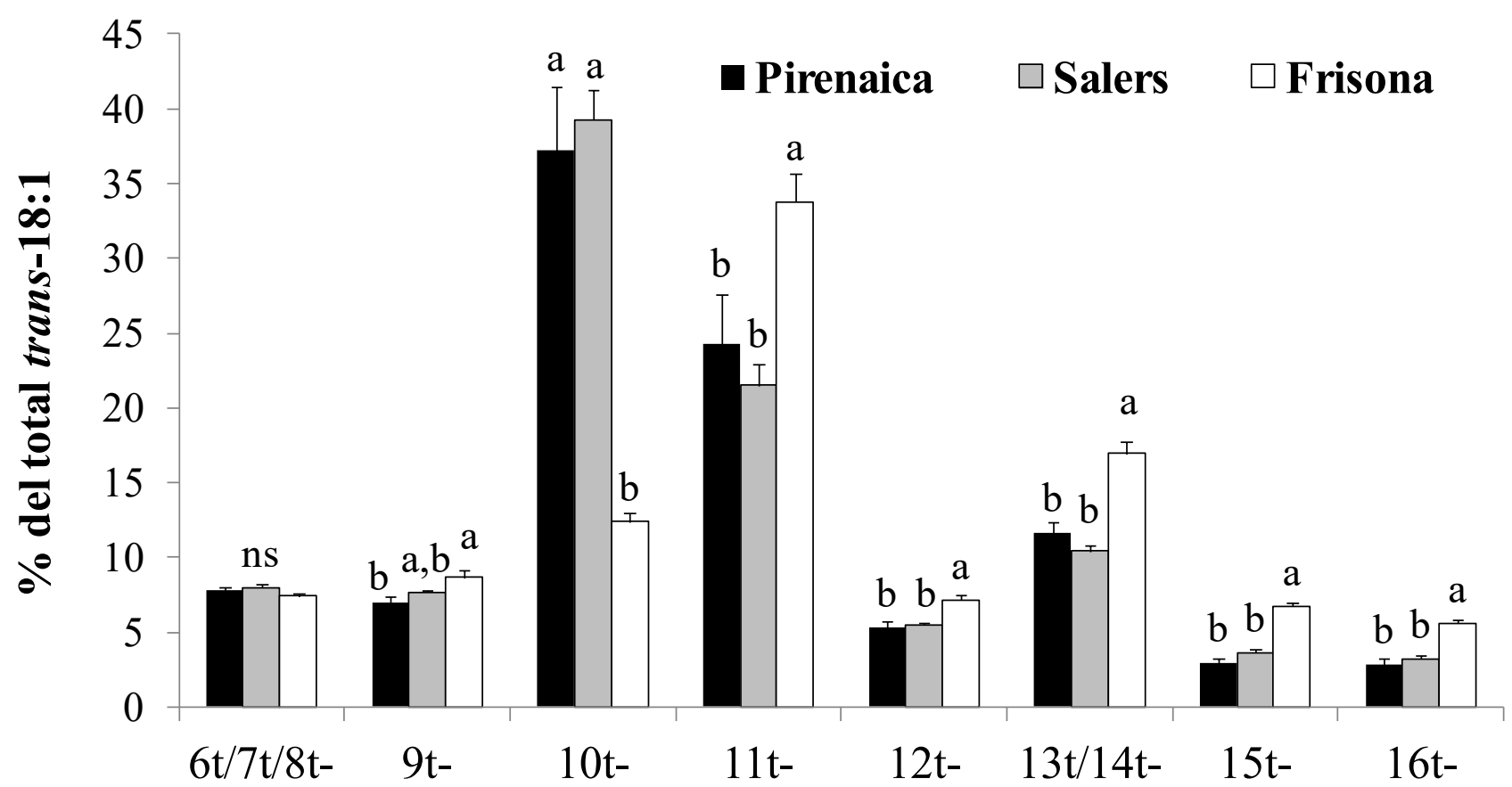

Figura 1. Abundancia relativa (\%) de los isómeros individuales trans-18:1 con respecto al contenido total de ácidos trans-18:1 en la grasa subcutánea de los tres grupos comerciales de vacuno: terneros de razas Pirenaica $(n=24)$ y Salers $(n=13)$; vacas de desvieje de raza Frisona $(n=24)$ en la Comunidad Autónoma del País Vasco (Relative abundance (\%) of individual trans-18:1 isomers in relation to the total trans-18:1 content in subcutaneous fat from the three bovine commercial types: calves from Pirenaica $(n=24)$ and Salers $(n=13)$ breeds; Friesian cull cows $(n=24)$ in the Basque Autonomous Community). a,b,cLetras diferentes indican diferencias significativas entre los grupos comerciales $(p<0,05)$; ns: no significativo.

El porcentaje de AGM tipo trans fue significativamente superior $(P<0,001)$ en la grasa SC de los terneros de raza Pirenaica $(6,37 \%)$ en comparación con los otros dos grupos comerciales bovinos (valor medio para ambos grupos de 4,19\%). Estas diferencias se observaron tanto para el total de trans-16:1 $(p<0,01)$ como de trans-18:1 ( $<<0,001)$. En cuanto a los isómeros trans-18:1, siendo los mayoritarios en la grasa de rumiantes, el contenido de 10t-18:1 fue significativamente mayor en la grasa SC de terneros de raza Pirenaica $(2,88 \%)$, intermedio para los terneros de Salers $(1,81 \%)$, y menor en la grasa de las vacas de desvieje $(0,437 \%$; $\mathrm{p}<0,001)$. Por otro lado, no se encontraron diferencias significativas entre los grupos comerciales bovinos para el porcentaje de $\mathrm{AV}$, aunque sí para la relación 10t/11t, la cual que fue significativamente superior $(p<0,001)$ en la grasa SC de los grupos de terneros (valor medio de 3,08 ) en comparación con la grasa SC de las vacas $(0,400)$. Según Bessa et al. (2015), una relación 10t/11t superior a 1 es indicativo de alteración o cambio en los procesos de biohidrogenación en el rumen a favor de la acumulación de 10t-18:1, lo cual ocurre, en general, cuando los animales son alimentados con alta proporción de alimento concentrado con o sin suplementación con aceites vegetales o semillas de oleaginosas. Si se compara el perfil de los isómeros trans-18:1 respecto al contenido total de trans-18:1 (figura 1), se observan claras diferencias entre los grupos comerciales bovinos estudiados. El isómero 10t-18:1 fue mayoritario en la grasa SC de los grupos de teneros en comparación con la grasa SC de las vacas de desvieje. El perfil isomérico de la grasa SC de los terneros de las razas Pirenaica y Salers fue muy similar al perfil previamente descrito por otros autores en animales jóvenes alimentados a base de concentrado (Dugan et al., 2008; Aldai et al., 2010; Rosa et al., 2014). En la literatura científica se ha descrito la formación de los isómeros trans-18:1 durante la biohidrogenación de los ácidos linolénico (ALN) y linoleico (AL) por acción de la microbiota ruminal (Harfoot y Hazlewood, 1997), así como la relación existente entre la abundancia de dichos isómeros con factores como la alimentación de los animales (ver revisiones bibliográficas de Aldai et al., 2011, 2013; Bessa et al., 2015). En la figura 1 también se puede observar que, en general, los isómeros trans-18:1 asociados al metabolismo del ALN, son significativamente mayores en el grupo de vacas de desvieje de raza Frisona.

No hubo diferencias en el contenido total de ALC entre los tres grupos comerciales bovinos (tabla II); el AR fue el isómero mayoritario en todos los grupos, seguido del ácido $7 t, 9 c-18: 2$. Se observaron diferencias significativas al representar los resultados como abundancia relativa de isómeros individuales linoleico conjugados con respecto al contenido total de ALC (figura 2). La abundancia relativa de $9 c, 11 t-, 11 t, 13 c$ - y el total de $t, t-18: 2$ fue significativamente mayor $(\mathrm{p}<0,05)$ en la grasa SC de las vacas de desvieje frente a la de los terneros, mientras que ocurrió lo contrario para los porcentajes de $7 t, 9 c-, 9 t, 11 c-$ y $10 t, 12 c-18: 2$. Dannenberger et al. (2005) encontraron concentraciones de $11 t, 13 c-18: 2$ hasta 15 veces mayores en terneros alimentados en régimen de pastoreo respecto a terneros alimentados a base de concentrado, así como un aumento significativo de algunos isómeros tipo $t, t-18: 2$.

El contenido total de AGP fue significativamente mayor en la grasa SC de los grupos de terneros de las razas Salers y Pirenaica (valor medio para ambas 


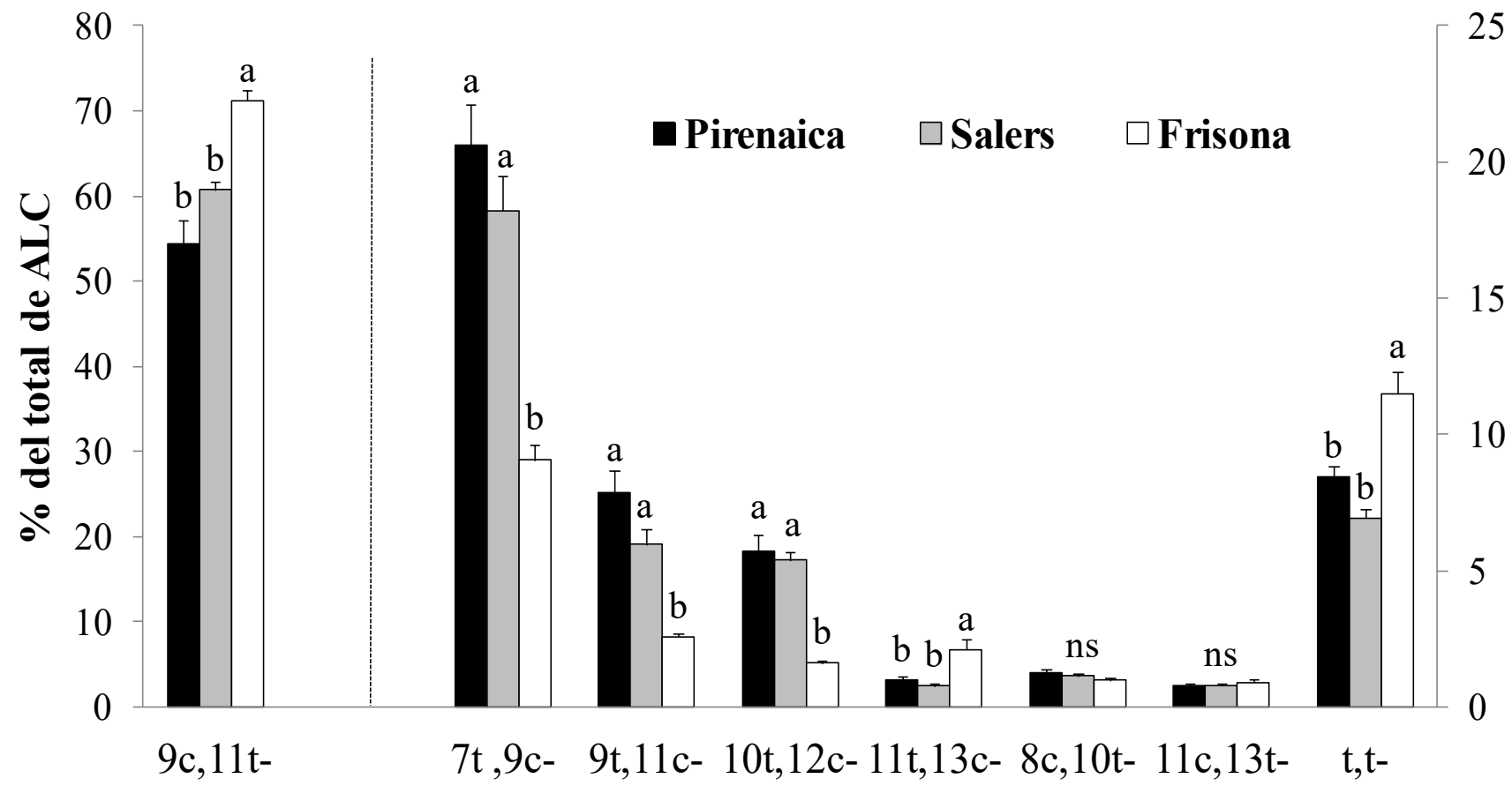

Figura 2. Abundancia relativa (\%) de los isómeros individuales del ácido linoleico conjugado (ALC) con respecto al contenido total de ALC en la grasa subcutánea de los tres grupos comerciales de vacuno: terneros de razas Pirenaica $(n=24)$ y Salers $(n=13)$; vacas de desvieje de raza Frisona $(n=24)$ en la Comunidad Autónoma del País Vasco (Relative abundance (\%) of individual conjugated linoleic acid (CLA) isomers in relation to the total CLA content in subcutaneous fat from the three bovine commercial types: calves from Pirenaica $(n=24)$ and Salers $(n=13)$ breeds; Friesian cull cows $(n=24)$ in the Basque Autonomous Community).

a,b,c Letras diferentes indican diferencias significativas entre los grupos comerciales $(p<0,05)$; ns, no significativo.

El contenido de $9 c, 11 t-18: 2$ se representa en el eje de la izquierda mientras que el del resto de los isómeros se representa en el eje de la derecha.

razas de 3,49\%), en comparación con la grasa SC de las vacas de desvieje $(1,81 \% ; p<0,001$; tabla II). Esta idea también ha sido observada en otros trabajos donde se ha comparado ganado vacuno de desvieje frente a vacuno jóven (Dugan et al., 2008). Estas diferencias fueron principalmente debidas a los contenidos de AGP tipo n-6 y, en particular, al AL que representó alrededor del $90 \%$ del total de los ácidos de tipo n-6. No se encontraron diferencias significativas en los AGP tipo n-3 entre los tres grupos comerciales bovinos (valor medio de 0,267\%). Sin embargo, la abundancia relativa de los ácidos n-3 respecto al total de AGP fue significativamente mayor $(p<0,001)$ en la grasa SC de las vacas $(15,6 \%)$ con respecto a los grupos de terneros $(8,42 \%$ y $6,92 \%$ para las razas Pirenaica y Salers, respectivamente). Por consiguiente, el grupo comercial de vacas de desvieje presentó una menor relación n-6/n-3 (5,94) en comparación con los grupos de terneros (valor medio para ambos grupos de 12,5; $\mathrm{p}<0,001$ ). De acuerdo al perfil de AG obtenido y según la literatura científica (Rosa et al., 2014), los terneros de raza Pirenaica y Salers reflejaban un alto consumo de concentrado en sus dietas, mientras que el perfil de las vacas de desvieje de raza Frisona puso de manifiesto el aporte de cierta cantidad de forraje y/o pasto en su alimentación. No obstante, es de destacar la posible influencia de la aptitud productiva, la edad de sacrificio o el estado de engrasamiento de los animales en la composición de la grasa.

\section{CONCLUSIONES}

Desde el punto de vista de la calidad nutricional, la grasa procedente de los terneros de las razas Pirenaica y Salers sacrificados en la Comunidad Autónoma del País Vasco presentó un menor contenido en ácidos grasos saturados a la vez que un mayor contenido en ácidos grasos de tipo n-6 y trans, como por ejemplo el 10t-18:1, 7t,9c-18:2, 9t,11c-18:2, y 10t,12c-18:2. En cambio, la grasa procedente de las vacas lecheras de desvieje de raza Frisona sacrificadas en la Comunidad Autónoma del País Vasco presentó un perfil con un mayor contenido en ácidos grasos de tipo n-3 y de metabolitos intermediarios saludables como los ácidos ruménico, vaccénico y 11t,13c-18:2. Estos resultados pueden contribuir a la valorización de la carne de vacas de desvieje de las explotaciones ganaderas de vacuno de leche de la Comunidad Autónoma del País Vasco desde el punto de vista de su comercialización, y por tanto, suponer un valor añadido para los ganaderos de esta comunidad autónoma.

\section{AGRADECIMIENTOS}

M. Aurtenetxe, X. Belaunzaran y D. Gamarra agradecen al Departamento de Desarrollo Económico y de Competitividad, y L. Bravo-Lamas al Departamento de Educación, Política Lingüística y Cultura, del Gobierno Vasco por las becas de formación. N. Aldai agradece al 
Ministerio de Economía y Competitividad y a la UPV / EHU por el contrato Ramón y Cajal (RYC-2011-08593). Este trabajo fue financiado por el Departamento de Educación, Universidades e Investigación del Gobierno Vasco (IT766-13, IT944-16). Los autores agradecen a J.P. Larrea y al personal del matadero de HarakaiUrkaiko por su colaboración y disponibilidad.

\section{BIBLIOGRAFÍA}

Albertí, P.; Ripoll, G.; Goyache, F.; Lahoz, F.; Olleta, J.L.; Panea, B. and Sañudo C. 2005. Carcass characterisation of seven Spanish beef breeds slaughtered at two commercial weights. MeatSci, 71:514-521.

Aldai, N.; Dugan, M.E.; Juárez, M.; Martínez, A. and Osoro, K. 2010. Double-muscling character influences the trans-18:1 and conjugated linoleic acid profiles in concentrate-fed yearling bulls. Meat Sci, 85: 59-65.

Aldai, N.; Dugan, M.; Kramer, J.; Martínez, A.; López-Campos, O.; Mantecón, A.R. and Osoro K. 2011. Length of concentrate finishing affects the fatty acid composition of grass-fed and genetically lean beef: an emphasis on trans-18: 1 and conjugated linoleic acid profiles. Animal, 5: 1643-1652.

Aldai, N.; de Renobales, M.; Barron, L.J.R. and Kramer, J.K.G. 2013. What are the trans fatty acids issues in foods after discontinuation of industrially produced trans fats? Ruminant products, vegetable oils, and synthetic supplements. Eur J Lipid Sci Techc, 115: 1378-1401.

Alves, S.P. and Bessa, R.J. 2009. Comparison of two gas-liquid chromatography columns for the analysis of fatty acids in ruminant meat. J Chromatogr A, 1216: 5130-5139.

Alves, S.P. and Bessa, R.J. 2014. The trans-10, cis-15 18:2: a missing intermediate of trans-10 shifted rumen biohydrogenation pathway? Lipids, 49: 527-541.

Bauchart, D.; Roy, A.; Lorenz, S.; Chardigny, J.M.; Ferlay, A.; Gruffat, D.; Sebedio, J.L.; Chilliard, Y. and Durand, D. 2007. Butters varying in trans 18:1 and cis-9, trans- 11 conjugated linoleic acid modify plasma lipoproteins in the hypercholesterolemic rabbit. Lipids, 42: 123-133.

Bazzoli, I.; De Marchi, M.; Cecchinato, A.; Berry, D.P and Bittante, G. 2014. Factors associated with age at slaughter and carcass weight, price, and value of dairy cull cows. J Dairy Sci, 97: 1082-1091.

Belaunzaran, X.; Bravo-Lamas, L.; Kramer, J.K.G. and Aldai, N. 2014. Limitation of using silver ion solid-phase extraction for animal lipids with a low trans content. Eur J Lipid Sci Tech, 116: 1621-1625.

Belaunzaran, X.; Bravo-Lamas, L.; Kramer, J.K.G.; Morales, R. and Aldai, N. 2016. Silver ion solid-phase extraction cartridges employing glass housings overcome the limitations observed in the GC analysis of animal lipids with low trans fatty acid content. Eur J Lipid Sci Tech (DOI: 10.1002/ejlt.201600124).

Bessa, R.J.; Alves, S.P.; Santos Silva, J. 2015. Constraints and potentials for the nutritional modulation of the fatty acid composition of ruminant meat. Eur J Lipid Sci Tech. 117: 1325-1344.

Bravo-Lamas, L.; Barron, L.J.R.; Kramer, J.K.G.; Etaio, I. and Aldai, N. 2016. Characterization of the fatty acid composition of lamb commercially avaiable in northern Spain: Emphasis on the trans-18:1 and CLA content and profile. Meat Sci, 117: 108-116.

Burnyeat, A. 2015. Brussels ponders EU limits on trans fats. http://www. globalmeatnews.com/Industry-Markets/Brussels-ponders-EU-limitson-trans-fats (7/12/2015).

Cabaraux, J.F.; Dufrasne, I.; Roux, M.; Istasse, L. et Hornick JL. 2005. La production de viande bovine a partir de femelles de reforme. INRA Prod Anim, 18: 37-48

Carballo, J.A. y Moreno, T. 2006. Características cuantitativas de las canales de vacas de desecho en Galicia. Arch Zootec, 55: 339-350.

Chilliard, Y.; Glasser, F.; Ferlay, A.; Bernard, L.; Rouel, J. and Doreau, M. 2007. Diet, rumen biohydrogenation and nutritional quality of cow and goat milk fat. Eur J Lipid Sci Tech, 109: 828-855.

Collins, J.M.; Neville, M.J.; Hoppa, M.B. and Frayn, KN. 2010. De novo lipogenesis and stearoyl-CoA desaturase are coordinately regulated in the human adipocyte and protect against palmitate-induced cell injury. J Biol Chem, 285: 6044-6052.

Cranwell, C.D.; Unruh, J.A.; Brethour, J.R.; Simms, D.D. and Campbell, R.E. 1996. Influence of steroid implants and concentrate feeding on performance and carcass composition of cull beef cows. J Anim Sci, 74: 1770-1776.

Cruz-Hernandez, C.; Deng, Z.; Zhou, J.; Hill, A.R.; Yurawecz, M.P.; Delmonte, P.; Mossoba, M.M.; Dugan, M.E.R. and Kramer, J.K.G. 2004. Methods for analysis of conjugated linoleic acids and trans-18:1 isomers in dairy fats by using a combination of gas chromatography, silver-ion thin-layer chromatography/gas chromatography, and silverion liquid chromatography. J AOAC Int, 87: 545-562.

Dannenberger, D.; Nuernberg, K.; Nuernberg, G.; Scollan, N.; Steinhart, $\mathrm{H}$. and Ender, K. 2005. Effect of pasture vs. concentrate diet on CLA isomer distribution in different tissue lipids of beef cattle. Lipids, 40: 589-598.

Delmonte, P.; Fardin-Kia, A.R.; Kramer, J.K.G.; Mossoba, M.M.; Sidisky, L. and Rader, J.I. 2011 . Separation characteristics of fatty acid methyl esters using SLB-IL1 11 , a new ionic liquid coated capillary gas chromatographic column. J Chromatogr A, 1218: 545-554.

Doreau, M. and Ferlay, A. 1995. Effect of dietary lipids on nitrogen metabolism in the rumen: a review. Livest Prod Sci, 43: 97-110.

Downs, S.M.; Thow, A.M. and Leeder, S.R. 2013. The effectiveness of policies for reducing dietary trans fat: A systematic review of the evidence. Bull World Health Organ, 91: 262-269.

Dugan, M.E.R.; Rolland, D.C.; Aalhus, J.L.; Aldai, N. and Kramer J.K.G. 2008. Subcutaneous fat composition of youthful and mature Canadian beef: emphasis on individual conjugated linoleic acid and trans-18:1 isomers. Can J Anim Sci, 88: 591-599.

Fievez, V.; Colman, E.; Castro-Montoya, J.M.; Stefanov, I. and Vlaeminck, B. 2012. Milk odd-and branched-chain fatty acids as biomarkers of rumen function - An update. Animal Feed Sci Tech, 172: 51-65.

Fritsche, S.; Rumsey, T.S.; Yurawecz, M.P.; Ku, Y. and Fritsche, J. 2001. Influence of growth promoting implants on fatty acid composition including conjugated linoleic acid isomers in beef fat. Eur Food Res Technol, 212: 621-629.

Glasser, F.; Schmidely, P.; Sauvant, D. and Doreau, M. 2008. Digestion of fatty acids in ruminants: a meta-analysis of flows and variation factors: 2. C18 fatty acids. Animal, 2: 691-704.

Harfoot, C.G. and Hazlewood, G.P. 1997. The Rumen microbial ecosystem. $2^{\text {nd }}$ ed. Chapman \& Hall. London, UK. pp: 382-426.

Jerónimo, E.; Alves, S.P.;Alfaia, C.M.; Prates, J.A.M.; Santos-Silva, J. and Bessa, R.J.B. 2011 . Biohydrogenation intermediates are differentially deposited between polar and neutral intramuscular lipids of lambs. Eur J Lipid Sci Tech, 113: 924-934.

Jurie, C.; Picard, B.; Hocquette, J.; Dransfield, E.; Micol, D. and Listrat, A. 2007. Muscle and meat quality characteristics of Holstein and Salers cull cows. Meat Sci, 77: 459-466.

Kennedy, P.M.; Young, B.A. and Christopherson, R.J. 1977. Studies on the relationship between thyroid function, cold acclimation and retention time of digesta in sheep. J Anim Sci, 45: 1084-1090.

Kramer, J.K.G.; Fellner, V.; Dugan, M.E.R.; Sauer, F.D.; Mossoba, M.M. and Yurawecz, M.P. 1997. Evaluating acid and base catalysts in the methylation of milk and rumen fatty acids with special emphasis on conjugated dienes and total trans fatty acids. Lipids, 32: 1219-1228.

Kramer, J.K.G.; Hernandez, M.; Cruz-Hernandez, C.; Kraft, J. and Dugan, M.E.R. 2008. Combining results of two $G C$ separations partly achieves determination of all cis and trans 16:1, 18:1, 18:2 and 18:3 except CLA isomers of milk fat as demonstrated using Ag-ion SPE fractionation. Lipids, 43: 259-273.

Lizaso, G.; Beriain, M.J.; Horcada, A.; Chasco, J. and Purroy, A. 2011. Effect of intended purpose (dairy/beef production) on beef quality. Can J Anim Sci, 91: 97-102.

Matulis, R,J.; Mckeith, F.K.; Faulkner, D.B.; Berger, L.L. and George, P. 1987. Growth and carcass characteristics of cull cows after different times-on-feed. J Anim Sci, 65: 669-674. 
Mozaffarian, D.; Aro, A. and Willett, WC. 2009. Health effects of trans-fatty acids: Experimental and observational evidence. Eur J Clin Nutr, 63: S5-S21.

BOE. 2008. Ministerio de Agricultura, Pescay Alimentación. Real Decreto 225/2008, de 15 de febrero, por el que se completa la aplicación del modelo comunitario de clasificación de las canales de vacuno pesado y se regula el registro de los precios de mercado. Núm. 56, de 5 de marzo de 2008. pp. 13309-13319.

Rosa, H.J.D.; Rego, O.A.; Silva, C.C.G.; Alves, S.P.; Alfaia, C.M.M.; Prates, J.A.M. and Bessa, R.J.B. 2014. Effect of corn supplementation of grass finishing of Holstein bulls on fatty acid composition of meat lipids. J Anim Sci, 92: 1-14.
Roy, A.; Chardigny, J.M.; Bauchart, D.; Ferlay, A.; Lorenz, S.; Durand, D.; Gruffat, D.; Faulconnier, Y.; Sebedio, J.L. and Chilliard, Y. 2007. Butters rich either in trans-10-C18:1 or in trans-11-C18:1 plus cis-9, trans- 11 CLA differentially affect plasma lipids and aortic fatty streak in experimental atherosclerosis in rabbits. Animal, 2007, 1: 467-476.

Smith, B.K.; Robinson, L.E.; Nam, R. and Ma, D.W.L. 2009. Trans-fatty acids and cancer: A mini-review. Brit J Nutr, 102: 1254-1266.

Wood, J.D.; Enser, M.; Fisher, A.V.; Nute, G.R.; Sheard, P.R.; Richardson, R.I.; Hughes, S.I. and Whittington, FM. 2008. Fat deposition, fatty acid composition and meat quality: A review. Meat Sci, 78: 343-358. 